\title{
Bayesian Unsupervised Learning with Multiple Data Types
}

\author{
Phaedra Agius, Yiming Ying and Colin Campbell \\ Department of Engineering Mathematics, Queen's Building, \\ University of Bristol, Bristol BS8 1TR \\ United Kingdom
}

\begin{abstract}
We propose Bayesian generative models for unsupervised learning with two types of data and an assumed dependency of one type of data on the other. We consider two algorithmic approaches, based on a correspondence model where latent variables are shared across datasets. These models indicate the appropriate number of clusters in addition to indicating relevant features in both types of data. We evaluate the model on artificially created data. We then apply the method to a breast cancer dataset consisting of gene expression and microRNA array data derived from the same patients. We assume dependence of gene expression on microRNA expression in this study. The method ranks genes within subtypes which have statistically significant abnormal expression and ranks associated abnormally expressing microRNA. We report a genetic signature for the basal-like subtype of breast cancer found across a number of previous gene expression array studies. Using the two algorithmic approaches we find that this signature also arises from clustering on the microRNA expression data and appears derivative from this data.
\end{abstract}

\section{Introduction}

Rapid developments in genomics and proteomics have lead to the generation of many different types of data which has in turn stimulated the development of data fusion techniques. Thus, for supervised learning, a number of different kernel-based methods have been proposed which enable class assignment based on the use of disparate types of input data. Successful multiple kernel classification methods have been proposed which use Bayesian methods [15], semi-definite programming [19], semi-infinite linear programming [26] and column generation methods [4], for example. In a bioinformatics context, examples have been presented where the classification test error is demonstrably reduced through the use of multiple types of data, encoded in different kernels, over the best single data type [14].

Though less investigated, unsupervised learning could be performed using multiple types of data in certain contexts. In this paper we propose a Bayesian unsupervised method for the joint modelling of two types of data which have an assumed dependency. The correspondence model 
we propose is inspired by correspondence $L D A$ (Latent Dirichlet Allocation [5, 6]), originally proposed for the joint modelling of images and their corresponding caption words. We propose two algorithmic approaches which we call corr $M L$ and $\operatorname{corr} V B$. In the experimental Section 3 we first evaluate performance on artificially created datasets with known labelling. This enables objective assessment of performance using a Jaccard score. We then investigate a breast cancer study in which microRNA and gene expression array datasets have been derived from the same patients. We assume there is a directed dependence of gene expression, at least in part, on microRNA expression. The resultant model is consistent with previous findings and biologically plausible. An important aspect of the proposed model is that we can establish the appropriate model complexity, the number of clusters in the data. In addition the model generates density estimates for the data belonging to the two component datasets.

\section{Bayesian Models and Inference}

Before describing the model we first introduce some notation: let $d$ denote the sample index and $D$ the corresponding number of samples. After training, the method represents samples as a combinatorial mixture over a finite set of soft clusters, with a probabilistic measure given for assignment of sample $d$ to cluster $k$. We consider two component datasets which we will label $C$ and $E$ and we will later assume dependence of $E$ on $C$. We will use $h$ and $g$ to label the respective features within datasets $C$ and $E$ respectively, with $H$ and $G$ denoting the corresponding number of features. Thus, for our breast cancer example in Section 3.2, these features are the labelled microRNA and genes respectively.

\subsection{A Correspondence Model for the joint modelling of two datasets}

In this section we introduce a correspondence model to capture an underlying functional interaction between component data sets. In line with previous models such as correspondence LDA [5], the two data sets are assumed to share a common prior distribution and latent variables. The correspondence model is applicable to the joint modelling of multiple datasets where there is a directed dependence of one type of data on another. In Section 3.2 we illustrate the model with a dataset for breast cancer, where we assume gene expression data (denoted $E$ ) is potentially dependent on microRNA data $($ denoted $C$ ): we will make reference to this example in our following discussion of the method to illustate the approach. Thus in this example we have pairs of samples $\left(C_{d}, E_{d}\right)$, i.e. both these readings are taken from the same patient, denoted sample $d$. We first generate a microRNA-specific measurement $C_{h d}$. A gene-specific measurement $E_{g d}$ is then generated conditioned on the generated cluster for microRNA sample $C_{d}$. The correspondence function is realized by a latent variable $y_{d g} \in[1, H]$ modeling the interaction between gene expression and microRNA measurements. This probabilistic graphical model is represented in Figure 1. 


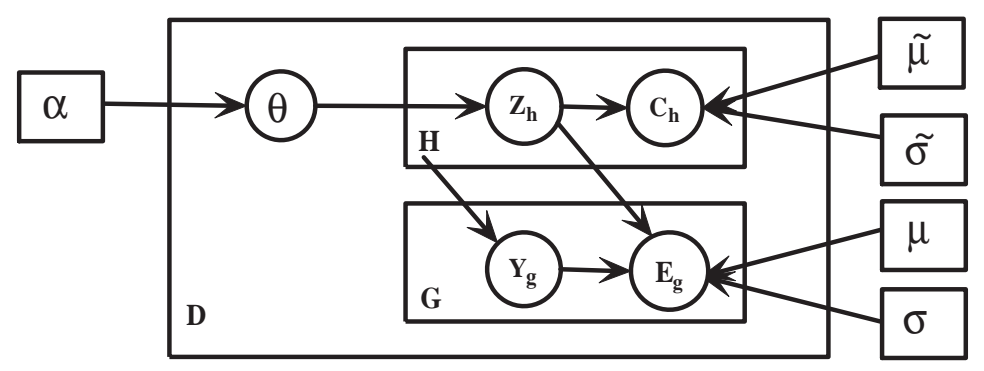

Figure 1: A graphical representation of the generative correspondence model. $C_{h d}$ and $E_{g d}$ are experimental observations and $\{\alpha, \mu, \sigma, \tilde{\mu}, \tilde{\sigma}\}$ are model parameters.

The model is described as follows:

\section{For a given data index $d$ for both $E(G \times D$ matrix $)$ and $C(H \times D$ matrix $)$}

1. Prior distributions: $\theta_{d} \sim \operatorname{Dir}_{K}(\alpha)$

2. Choose $C_{d}$ :

(a) Choose cluster for $C_{h d}$ : $z_{d h} \sim \operatorname{Multi}\left(\theta_{d}\right)$

(b) Sample $C_{h d} \sim \mathcal{N}\left(C_{h d} \mid \tilde{\mu}_{h z_{d h}}, \tilde{\sigma}_{h z_{d h}}\right)$ where $\mathcal{N}\left(C_{h d} \mid \tilde{\mu}, \tilde{\sigma}^{2}\right)$ denotes a normal distribution with mean $\tilde{\mu}$ and variance $\tilde{\sigma}^{2}$.

3. Choose $E_{d}$ :

(a) Sample gene correspondence: $y_{d g} \sim \operatorname{Uniform}(1, \ldots, H)$

(b) Sample $E_{g d} \sim \mathcal{N}\left(E_{g d} \mid \mu, \sigma, z, y_{d g}\right)=\mathcal{N}\left(E_{g d} \mid \mu_{g z_{d h}}, \sigma_{g z_{d h}}^{2}, y_{d g}=h\right)$

Using the notation $\Theta=\{\alpha, \mu, \sigma, \tilde{\mu}, \tilde{\sigma}\}$, the joint distribution for a given index $d$ is then specified by

$$
\begin{aligned}
p\left(C_{d}, E_{d}, z_{d}, y_{d}, \theta_{d} \mid \Theta\right) & =p\left(\theta_{d} \mid \alpha\right) \prod_{h}\left[p\left(z_{d h} \mid \theta_{d}\right) \mathcal{N}\left(C_{h d} \mid \tilde{\mu}_{h z_{d h}}, \tilde{\sigma}_{h z_{d h}}\right)\right] \\
& \times \prod_{g} p\left(y_{d g} \mid H\right) \mathcal{N}\left(E_{g d} \mid \mu_{g z_{d h}}, \sigma_{g z_{d h}}^{2}, y_{d g}=h\right)
\end{aligned}
$$


and the overall joint distribution is given by

$$
p(C, E, z, y, \theta \mid \Theta)=\prod_{d} p\left(C_{d}, E_{d}, z_{d}, y_{d}, \theta_{d} \mid \Theta\right)
$$

Extensions to classical Gaussian mixture models (GMM) (e.g. [28]) are possible to handle multiple data sets in a similar fashion to the mixture model presented here. However, for a GMM each datapoint $E_{d}$ is only related to a latent variable $z_{d}$ : this restricts the datapoint to an association with one cluster only. In contrast, as with Latent Process Decomposition [24] and several other soft cluster models, in our model each data point $C_{d}$ is associated with multiple latent variables $\left\{z_{d h}: h \in[1, \ldots, H]\right\}$. This means there is no implicit mutual exclusion of clusters assumption and $C_{d}$ can be associated with multiple clusters. As a correspondence model this also means the data $E_{d}$ can stochastically share $C_{d}$ clusters through the correspondence latent variable $y_{d g, h}$.

\subsection{CorrML: a maximum likelihood approach}

Having introduced the models we now focus on approximation inference and parameter estimation for the correspondence model. Let the overall set of latent variables be denoted by $\mathcal{H}=\{\theta, z, y\}$ and model parameters by $\Theta=\{\alpha, \mu, \sigma, \widetilde{\mu}, \widetilde{\sigma}\}$. Then the target of model inference is to compute the posterior distribution $p(\mathcal{H} \mid E, C, \Theta):=p(E, C, \mathcal{H} \mid \Theta) / p(E, C \mid \Theta)$ and to learn the model parameters $\Theta$. Unfortunately, this would involve computationally intensive estimation of the integral in the evidence $p(E, C \mid \Theta)$ and thus we will use variational inference instead [16] (we will discuss MCMC methods in the Conclusion).

The goal of variational inference is essentially to minimize the KL-divergence between the variational distribution $q(\theta, z, y)$ and posterior distribution $p(E, C, \theta, z, y \mid \Theta)$ :

$$
\arg \min _{\Theta, q \in \mathcal{Q}} \operatorname{KL}(q(\theta, z, y) \| p(E, C, \theta, z, y \mid \Theta))
$$

Since this expression is not convex, we employ the mean field approach [16]. The derivations are standard (see Jordan et al [16]) and referred to as variational EM-steps.

We will briefly describe the general methodology. For simplicity, we assume that the latent variables $\mathcal{H}$ can be split into sub-variables $\mathcal{H}_{i}$. Then we choose the hypothesis family $\mathcal{Q}$ of variational distributions $q(\mathcal{H})$ to be a fully factorized family, that is, $q(\mathcal{H}):=\prod_{i} q\left(\mathcal{H}_{i}\right)$. Consequently, for the variational E-step, we conclude that the variational distribution of latent variables is given by $[16,5]$ :

$$
q\left(\mathcal{H}_{i}\right) \propto \exp \left(\mathbf{E}_{q \backslash i}[p(E, \mathcal{H} \mid \Theta)]\right)
$$

where $q^{\backslash i}$ represents the distribution $\prod_{j \neq i} q\left(\mathcal{H}_{j}\right)$. For the M-Step, we take the derivative of the KL-divergence with respective to model parameter $\Theta$ and obtain the updates for $\Theta$. 
We now apply the above methodology to the correspondence model and obtain the following update equations. Let $\mathcal{H}=\{z, \theta, y\}$ and assume that the family of variational distributions $\mathcal{Q}$ takes the form:

$$
q(\theta, z, y)=\left[\prod_{d} q\left(\theta_{d} \mid \gamma_{d}\right)\right]\left[\prod_{d, h} q\left(z_{d h} \mid R_{d h}\right)\right]\left[\prod_{d, g} q\left(y_{d g} \mid Q_{d g}\right)\right],
$$

where $q\left(\theta_{d} \mid \gamma_{d}\right)$ is a Dirichlet distribution, $q\left(z_{d h} \mid R_{d h}\right)$ and $\prod_{d g} q\left(y_{d g} \mid Q_{d g}\right)$ are multinomial distributions. $\gamma, R, Q$ are often called variational parameters and describe sufficient statistics of the variational distributions $q$. Equation (11) tells us that the optimal $q$ can be found via the updates:

$$
\begin{aligned}
q(\theta \mid \gamma) & =\prod_{d} q\left(\theta_{d} \mid \gamma_{d}\right) \propto \mathbf{E}_{z, y}[\log p(E, C, \theta, z, y \mid \Theta)], \\
q(z \mid R) & =\prod_{d, h} q\left(z_{d h} \mid R_{d h}\right) \propto \mathbf{E}_{\theta, y}[\log p(E, C, \theta, z, y \mid \Theta)], \\
q(y \mid Q) & =\prod_{d, g} q\left(y_{d g} \mid Q_{d g}\right) \propto \mathbf{E}_{\theta, z}[\log p(E, C, \theta, z, y \mid \Theta)] .
\end{aligned}
$$

In summary, the estimation of the $\log$ of the joint distribution yields variational $E M$-type updates for variational and model parameters, as follows:

- Variational E-step:

$$
\begin{gathered}
\gamma_{d k}=\alpha_{k}+\sum_{h} R_{d h k} \\
R_{d h k} \propto \mathcal{N}\left(C_{h d} \mid \tilde{\mu}_{h k}, \tilde{\sigma}_{h k}\right) \exp \left(\Psi\left(\gamma_{d k}\right)-\Psi\left(\sum_{j} \gamma_{d k}\right)+\sum_{g} Q_{d g h} \log \mathcal{N}\left(E_{g d} \mid \mu_{g k}, \sigma_{g k}^{2}\right)\right) \\
Q_{d g h} \propto \exp \left(\sum_{k} R_{d h k} \log \mathcal{N}\left(E_{g d} \mid \mu_{g k}, \sigma_{g k}^{2}\right)\right) .
\end{gathered}
$$

In the variational M-step we update the model parameters $\Theta$. To this end, we just take the derivatives of the KL-divergence. The updates are listed as follows:

- Variational $M$-step:

$$
\tilde{\mu}_{h k}=\frac{\sum_{d} R_{d h k} C_{h d}}{\sum_{d} R_{d h k}}, \quad \tilde{\sigma}_{h d}^{2}=\frac{\sum_{d} R_{d h k}\left(C_{h d}-\tilde{\mu}_{h k}\right)^{2}}{\sum_{d} R_{d h k}}
$$




$$
\mu_{g k}=\frac{\sum_{d, h} Q_{d g h} R_{d h k} E_{g d}}{\sum_{d, h} Q_{d g h} R_{d h k}}, \quad \sigma_{g k}^{2}=\frac{\sum_{d, h} Q_{d g h} R_{d h k}\left(E_{g d}-\mu_{g k}\right)^{2}}{\sum_{d, h} Q_{d g h} R_{d h k}}
$$

For the updates for $\alpha$, we use a Newton-Raphson method (see the Appendix of [6]). The gradient is given by: $\frac{\partial \mathcal{L}}{\partial \alpha_{i}}=D\left(\Psi\left(\sum_{k} \alpha_{k}\right)-\Psi\left(\alpha_{i}\right)\right)+\sum_{d}\left(\Psi\left(\gamma_{d i}\right)-\Psi\left(\sum_{k} \gamma_{d k}\right)\right)$, the Hessian is $H_{i j}=D\left(\Psi^{\prime}\left(\sum_{k} \alpha_{k}\right)-\delta_{i j} \Psi^{\prime}\left(\alpha_{i}\right)\right)$. Hence, we have an iterative update procedure:

$$
\alpha_{\text {new }}=\alpha_{\text {old }}-\left(H\left(\alpha_{\text {old }}\right)\right)^{-1} \frac{\partial \mathcal{L}\left(\alpha_{\text {old }}\right)}{\partial \alpha} .
$$

We pursue the above iterative procedure until convergence of the KL-divergence (details are given in the Appendix A: for discussion of numerical stability issues for the variational Estep update see Rogers et al [24] section 5.3). Since the latent variable $\theta_{d k}$ is the $k$-th cluster probability of the sample $d$ and its expectation with respective to the posterior distribution $q\left(\theta_{d}\right)$ is $\gamma_{d k}$, we can assign data $E_{d}$ and $C_{d}$ to cluster $k$ using $k^{*}=\arg \max _{k} \gamma_{d k}$, for example. In Section 3 , with a knowledge of the means and variances $\left(\mu, \sigma^{2}\right)$ and $\left(\tilde{\mu}, \tilde{\sigma}^{2}\right)$, we can use statistical scores to perform gene-ranking and thus find abnormally expressing genes or microRNA. Following our earlier practice [24], we can choose the appropriate number of clusters using cross-validation on the predictive likelihood (see Appendix A for details).

We end this subsection with some comments. The above method can also handle cases where some values $E_{g d}$ or $C_{h d}$ are missing by omitting corresponding contributions in the $M$-step updates and corresponding parameters $Q_{d g, k}$ and $R_{d g, h}$. In the original correspondence model of Blei et al [5] clustering was performed over samples. Here we are more interested in clustering over samples and trying to find a linkage between features (e.g. microRNA and genes). Unfortunately, whereas a direct linkage is calculable in the original correspondence model, $p\left(E_{g d} \mid C_{h d}\right)$ is not meaningfully calculable here. Thus the model proposed here gives a picture of altered features within each cluster but does not individually link these: such a direct linkage would require methods outside the algorithm such as correlation analysis.

\subsection{CorrVB: a variational Bayes approach}

In the maximum likelihhod approach above, a computationally expensive cross validation study is required to infer appropriate number of clusters. This involves setting aside a certain percentage of the data and then estimating the parameters on the remaining data. A model accuracy score is then found from the estimated likelihood on left-out data. This variational inference approach only gives a point estimate of $\{\mu, \sigma, \widetilde{\mu}, \widetilde{\sigma}\}$. An alternative variational inference is the variational Bayesian method which allows us to estimate the full posterior distribution in place of point estimates. Another advantage of a variational Bayesian approach over a maximum likelihood solution is that an inbuilt mechanism for model comparison can be performed more easily. 
We now turn our attention to the description of variational Bayesian inference for our correspondence model. To this end, we further regard $\Xi=\{\mu, \sigma, \widetilde{\mu}, \widetilde{\sigma}\}$ as latent variables. Specifically, we further assume their prior distributions as follows. Let

$$
p\left(\mu \mid m_{0}, v_{0}\right)=\prod_{g, k} \mathcal{N}\left(\mu_{g k} \mid m_{0}, v_{0}\right), \quad p\left(\widetilde{\mu} \mid m_{0}, v_{0}\right)=\prod_{h, k} \mathcal{N}\left(\widetilde{\mu}_{h k} \mid m_{0}, v_{0}\right),
$$

and

$$
p\left(\beta \mid a_{0}, b_{0}\right)=\prod_{g, k} \Gamma\left(\beta_{g k} \mid a_{0}, b_{0}\right), \quad p\left(\widetilde{\beta} \mid a_{0}, b_{0}\right)=\prod_{h, k} \Gamma\left(\widetilde{\beta}_{h k} \mid a_{0}, b_{0}\right)
$$

where the Gamma distribution is defined by $\Gamma\left(x \mid a_{0}, b_{0}\right)=x^{a_{0}-1} e^{-\frac{x}{b_{0}}} / \Gamma\left(a_{0}\right) b_{0}^{a_{0}}$.

For fixed $\alpha$, the variational Bayesian (ensemble learning) method (see e.g. [2]) aims to find the approximate posterior distribution $q \in \mathcal{Q}$ to the true posterior distribution $p(\theta, z, y, \Xi \mid E, \alpha)$ ), i.e.

$$
\left.\min _{q \in \mathcal{Q}} \operatorname{KL}(q(\theta, z, y, \Xi) \| p(\theta, z, y, \Xi \mid C, E, \alpha))\right) .
$$

Note, for any variational distribution $q(\theta, z, y, \Xi)$, that

$$
\begin{aligned}
\log p(E \mid \alpha) & =\log \int \sum_{Z} p(C, E, \theta, z, y, \Xi \mid \alpha) d \theta d z d y d \Xi \\
& \left.=\mathbb{E}_{q}\left[\log \frac{p(C, E, \theta, z, y, \Xi \mid \alpha)}{q(\theta, z, y, \Xi)}\right]+\operatorname{KL}(q(\theta, z, y, \Xi) \| p(\theta, z, y, \Xi \mid C, E, \alpha))\right) .
\end{aligned}
$$

Since $p(E)$ is a constant, our optimization target is equivalently reduced to maximizing the free-energy lower bound defined by

$$
\max _{q} \mathcal{F}_{\mathcal{K}}(q \mid \alpha):=\max _{q} \mathbb{E}_{q}\left[\log \frac{p(C, E, \theta, z, y, \Xi \mid \alpha)}{q(\theta, z, y, \Xi)}\right]
$$

If we have no restriction on variational distributions $q$, then the maximizer of the free energy bound is trivially the true posterior which is already assumed intractable. Hence, we should introduce the hypothesis family $\mathcal{Q}$ where the variational posterior distributions $q(\theta, Z, \Theta)$ live on. For simplicity, we assume that the overall latent variables $\mathcal{H}=\{\theta, z, y, \mu, \sigma, \widetilde{\mu}, \widetilde{\sigma}\}$ can be split into sub-variables $\mathcal{H}_{i}$. Then we choose the hypothesis family $\mathcal{Q}$ of variational distributions $q(\mathcal{H})$ to be a fully factorized family, that is, $q(\mathcal{H}):=\prod_{i} q\left(\mathcal{H}_{i}\right)$. Consequently, in analogy to the E-step in the ML inference the variational distribution of latent variables is given by $[16,2]$ :

$$
q\left(\mathcal{H}_{i}\right) \propto \exp \left(\mathbf{E}_{q^{\backslash i}}[\log p(C, E, \theta, z, y, \Xi \mid \alpha)]\right)
$$


where $q^{\backslash i}$ represents the distribution $\prod_{j \neq i} q\left(\mathcal{H}_{j}\right)$. Specifically, the variational posterior distribution can be represented by their corresponding variational parameters as follows.

$$
\begin{aligned}
q(\theta, z, y, \Xi)= & {\left[\prod_{d} q\left(\theta_{d} \mid \gamma_{d}\right)\right]\left[\prod_{d, h} q\left(z_{d h} \mid R_{d h}\right)\right]\left[\prod_{d, g} q\left(y_{d g} \mid Q_{d g}\right)\right] } \\
& \times\left[\prod_{h, k} q\left(\widetilde{\mu}_{h k} \mid \widetilde{m}_{h k}, \widetilde{v}_{h k}\right) q\left(\widetilde{\beta}_{h k} \mid \widetilde{a}_{h k}, \widetilde{b}_{h k}\right)\right] \\
& \times\left[\prod_{g, k} q\left(\mu_{g k} \mid m_{g k}, v_{g k}\right) q\left(\beta_{g k} \mid a_{g k}, b_{h k}\right)\right] .
\end{aligned}
$$

Since the Gamma distribution is the conjugate prior of the Normal distribution, the variational posterior distribution on the latent variables $\mu$ and $\beta$ are respectively Normal distribution and Gamma distribution and likewise for $\widetilde{\mu}$ and $\widetilde{\beta}$. The detailed updates are listed on Appendix B. We can also update the parameter $\alpha$ together with the latent variables $\mathcal{H}$ which is known as Maximum a Posteriori (MAP) of type II. This inference method can be regarded as a regularization formulation of ML since there is prior distributions on $\Xi$ instead of their point estimates in ML approach. Specifically, MAP of type II is an EM algorithm by maximizing the lower (free energy) bound of the $\log$ likelihood $\log p(E \mid \alpha)$ with respect to both the latent variables and $\alpha$ :

$$
\max _{q, \alpha} \mathcal{F}_{\mathcal{K}}(q \mid \alpha):=\max _{q, \alpha} \mathbb{E}_{q}\left[\log \frac{p(C, E, \theta, z, y, \Xi \mid \alpha)}{q(\theta, z, y, \Xi)}\right] .
$$

As in the EM updates for ML solution, the updates for the latent variables $\mathcal{H}$ is called E-step. In the M-step, for fixed variational posterior distribution $q$, we can use Newton-Raphson method to update it by

$$
\alpha_{\text {new }}=\arg \max _{\alpha} \mathcal{F}(q \mid \alpha) .
$$

where, similar to the ML method, the updates for $\alpha$ can be solved by the Newton-Raphson method $\alpha_{\text {new }}=\alpha_{\text {old }}-\left(H\left(\alpha_{\text {old }}\right)\right)^{-1} \frac{\partial \mathcal{F}\left(q \mid \alpha^{\text {old }}\right)}{\partial \alpha}$.

\section{Experiments}

In this section we will numerically validate the proposed model. First we demonstrate that these models perform as expected on artificially generated data, where the cluster structure and sample labels are known. In addition, in Section 3.1, we consider an expression array dataset for S. Cerevisiae which illustrates a biological context in which correspondence models would be relevant. We compare against three other clustering methods. We then consider the breast cancer example referred to earlier where gene expression array data is assumed dependent on microRNA data. The results for CorrVB validate the results for CorrML and the results are consistent with previous studies. 


\subsection{Comparison with other clustering methods}

To validate performance we first generated artificial datasets. Data for $C$ was randomly generated to give three distinct clusters (consisting of 10 samples per cluster with 10 features per sample). Then the data in $E$ was generated per sample in $C$, so that it had blocks of features positively correlated to features in $C$. The number of features in $E$ was varied between $N=2, \ldots, 10$ times the size of dataset $C$. Thus each vector in $C$ had from 2 to 10 replicate features in $E$ with each such feature perturbed by a small Gaussian random deviate addition to the corresponding feature value in $C$. Since the sample labels of our artificially generated data were known, we were able to use the Jacard score to compare our clusterings with the correct labels and thereby validate our results. The Jaccard score $J$ is used to compare clusterings, or to compare a clustering with the correct labels. If we let $n_{11}$ denote the number of point pairs correctly placed together in the clustering, $n_{01}$ the number of incorrectly identified pairs and $n_{10}$ the number of missed pairs, then $J=n_{11} /\left(n_{11}+n_{01}+n_{10}\right)$, where $0 \leq J \leq 1$, with 1 indicating perfectly correct clustering. In Figure 2 we present a bar plot of the Jaccard scores. Apart from the proposed correspondence model, we also amalgamated $C$ and $E$ and performed spectral clustering and $k$-means clustering on the amalgamated dataset. We also evaluated a novel joint mixture model (JMM), outlined in Appendix C, on this amalgamated dataset. To create this amalgamated dataset, both datasets were normalised to zero mean, unit variance and combined into a single column vector per sample. The corresponding Jaccard scores are given in Figure 2. All of the models perform better for a small $N$, with the correspondence model (corrML) consistently outperforming the rest. As $N$ increases, the difference in Jaccard scores diminishes considerably as it is hard for any of the models to pick up the correct clustering.
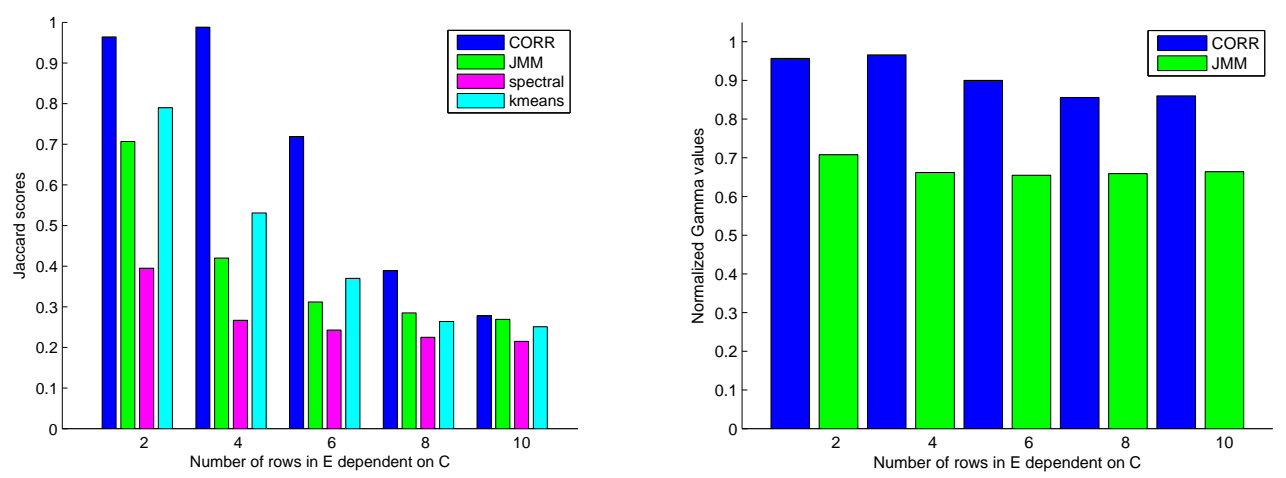

Figure 2: Average Jaccard scores on the 3 cluster artifical dataset (left) and associated confidence measures (right). CORR is the CorrML algorithm and JMM a joint mixture model outlined in Appendix C. 
We use the joint mixture model as comparator because, like the correspondence model, a normalised $\gamma_{d k}$ values (normalised over $k$ ), can represent the confidence in the assignment of sample $d$ to cluster $k$. In Figure 2 we show bar plots of for these confidence measures for both corrML and JMM. The values for the correspondence model are consistently higher for the correspondence model.

As a second example, we used microarray expression data for $S$. cerevisiae from a series of experiments run by Middendorf et al [22]. These authors identified a strong regulating factor, USV1, which was believed to influence up to 305 other genes in the dataset. In this second example, the $C$ data now consists of only one gene, USV1, while $E$ comprises the 305 regulated genes. Though an extreme example, since $C$ only has one value per sample, this is a context where the application of a correspondence model makes sense since the significance of $C$ is maintained by this model, but would be lost if we amalgamated the datasets, for example.

The samples were derived from three groups of experiments. The first group corresponded to a set of 7 heat shock experiments over various time intervals from 0 to 60 minutes. The second group corresponded to a set of 5 nitrogen depletion experiments over various time intervals from 12 hours to 5 days and the third group was a set of stationary phase experiments that were used as a time-zero reference. A PCA plot suggested these were reasonably well defined groupings. We ran the correspondence and joint mixture models and they correctly classified all of these groups $(J=1$, based on the highest predictive log-likelihood solution after 30 random initialisations). We also used $k$-means clustering and spectral clustering on the amalgamated dataset. Both $k$-means clustering and spectral clustering can give different results depending on the start point, hence we investigated performance over 100 restarts. $k$-means clustering gave $J=1$ with 62 restarts from the 100 with an overall average Jaccard score of 0.81 . Spectral clustering correctly classified $(J=1) 81$ from 100 restarts with an average Jaccard score of 0.90 . These results are not that surprising since $C$ is considerably smaller than $E$ in size so the significance of $C$ is lost when the two datasets are amalgamated together.

\subsection{Evaluation on a real-life dataset: breast cancer}

For the two examples given above we have argued that there are instances where joint modelling of the data is more appropriate than clustering on an amalgamated dataset. We now extend the discussion to a real-life example in cancer biology to illustrate the extra biological insights provided by the correspondence model. We will show that the results which emerge are consistent with previous findings. In addition, we have not commented so far on model complexity: how many clusters are present in the data. We will show that the estimated log-likelihood on hold-out data provides a principled approach to finding the correct model complexity.

We applied our models to a dataset consisting of two types of data derived from the same patients. The first data set, $C$, consisted of microRNA expression data from 78 primary human breast tumors using a bead-based array to identify 133 microRNA found in normal and breast tumors [7]. The second set of data, $E$, comprised gene expression data for the same 78 patients. 
In both cases, the data was normalized to zero mean and unit variance.

The first goal was to determine the optimal number of clusters. To do so, we first performed a cross validation study on the predictive log likelihood using CorrML (see Appendix A). We held out 8 datapoints as test data and the remaining 70 datapoints were used to construct the model: performance was averaged over 10 random partitionings of the data into training and test data. The log-likelihood on the hold-out data was calculated using the model obtained from training data. Figure 3 (left) shows the corresponding log-likelihood curve for the correspondence mode. A 5 cluster model appears optimal: if more than 5 clusters are used overfitting occurs and the log-likelihood falls. To confirm this result we then used the variational Bayes method of section 2.3. In this approach, we do not need hold-out data to estimate a log-likelihood. Instead, a free energy expression is used. In 3 (right) we give the corresponding curve for the free energy which likewise gives a peak at 5 clusters indicating that there are at least 5 principal subtypes of breast cancer.
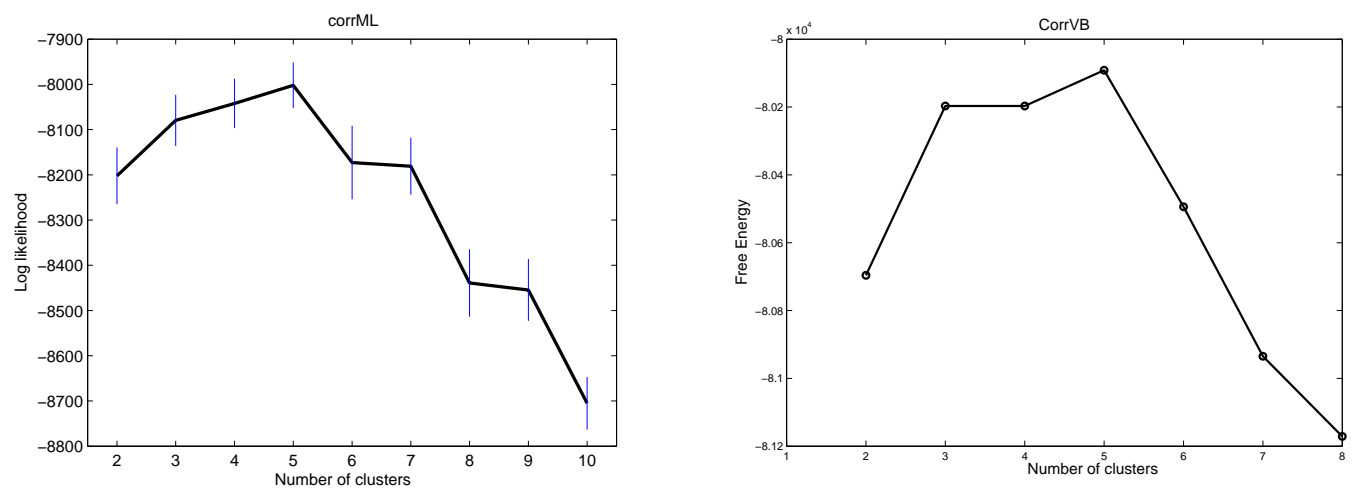

Figure 3: Log Likelihood versus number of clusters for maximum likelihood (left) and variational Bayes correspondence models (right).

As remarked in section 2.2 we can assign sample $d$ to cluster $k$ using $k^{*}=\arg \max _{k} \gamma_{d k}$. Based on available survival data, we can therefore derive Kaplan Meier plots for the 5 indicated subtypes. These are given in Figure 4. 

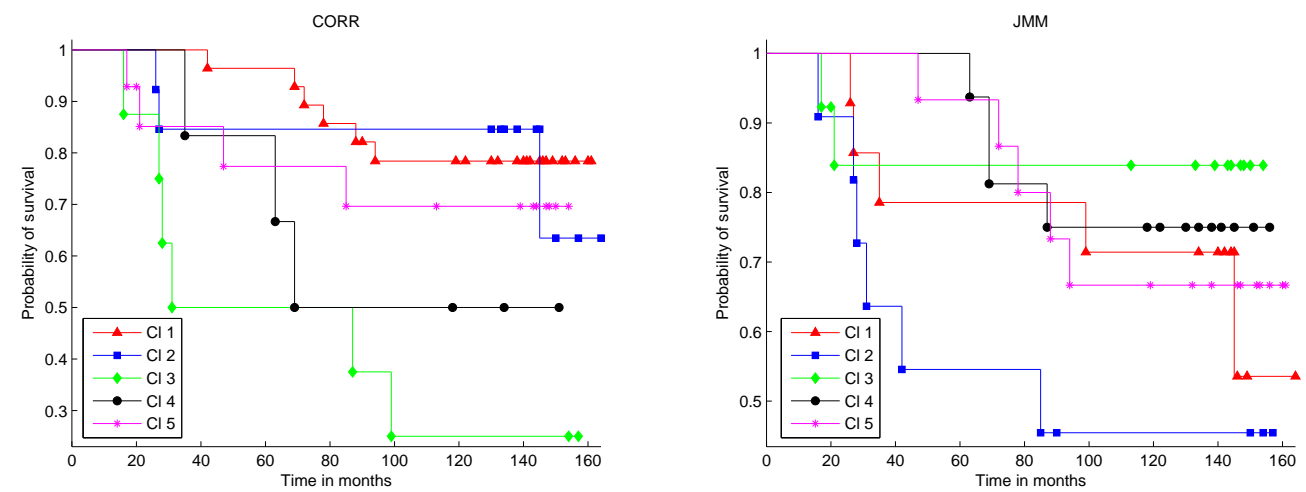

Figure 4: Kaplan Meier plots for correspondence (CorrML) and joint mixture models (amalgamated data).

We can also derive density estimates, quantifying the distribution of gene expression data values within subtypes. Using the correspondence model and the mean $\mu_{g k}$ and standard deviation $\sigma_{g k}$ of a gene $g$ within cluster $k$, we present the density distributions for some genes in Figure 5. FOXA1 and FOXC1 have very distinctive distributions for the subtype labelled Cl5: while FOXA1 underexpresses, FOXC1 is overexpresses this subtype. ERBB2 and GRB7 overexpress in subtype $C l 3$ : there is a well documented $E R B B 2+$ subtype of breast cancer [27]. 

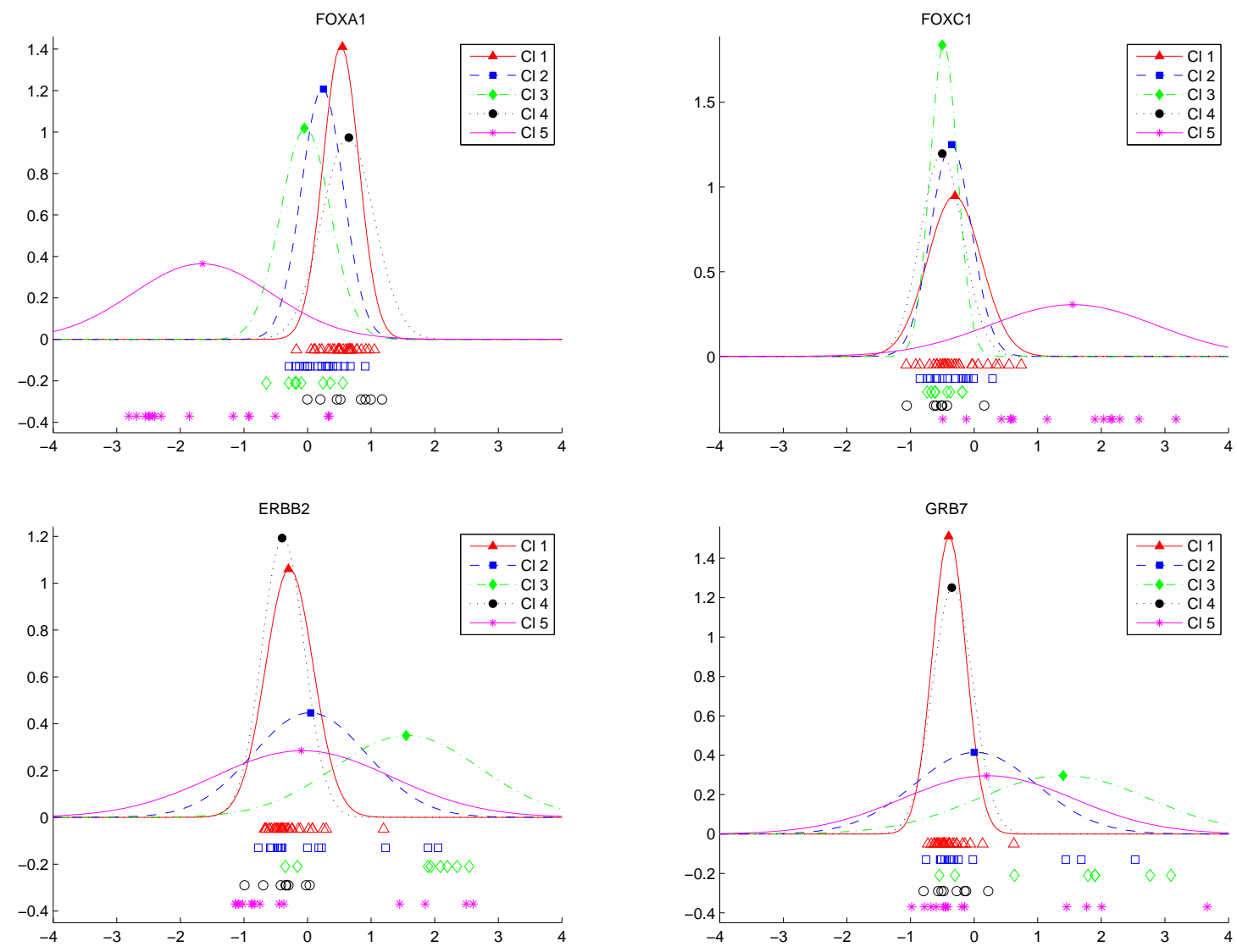

Figure 5: Density distribution plots for four genes using CorrML. Gene expression values are given at the base of each plot. A sample $d$ is assigned to the cluster $k$ depending on the largest value of the confidence measure, $\gamma_{d k}$. The Gaussian distributions are derived from $\left(\mu_{g k}, \sigma_{g k}\right)$ in equations 8 .

Abnormally expressed genes can be identified using a Fisher score $\left|\mu_{g_{1} k}-\mu_{g_{2} k}\right| / \sqrt{\left.\sigma_{g_{1} k}^{2}+\sigma_{g_{2} k}^{2}\right)}$, for example. However, this score tends to overlook genes with large spreads such as FOXC1 in Cl5 of Figure 5. Thus, we used a rank-based Mann-Whitney score instead to find genes abnormally expressing within one subtype relative to the other subtypes. In Table 1 we list the 20 top-ranked genes by significance for the 5 subtypes resolved by the correspondence model. 


\begin{tabular}{|c|c|c|c|c|}
\hline Cl 1 & Cl 2 & Cl 5 & Cl 4 & Cl 3 \\
\hline UBE2C & COL11A1 & GATA3 & CTGF & GSDML \\
\hline CDC20 & TIMP3 & FOXC1 & RARRES1 & ORMDL3 \\
\hline POSTN & AEBP1 & STARD10 & C1S & ERBB2 \\
\hline CYBRD1 & COL10A1 & MLPH & PRKACB & STARD3 \\
\hline OGN & PLAU & TOB1 & FBLN2 & FGFR4 \\
\hline$A D H 1 B$ & MFAP5 & AGR2 & TNC & ESR1 \\
\hline$A D H 1 A$ & COL12A1 & FBP1 & ACTA2 & PERLD1 \\
\hline CYP4X1 & MMP11 & GPR160 & CR598488 & CTXN1 \\
\hline COL10A1 & FN1 & C10orf116 & COL6A1 & DQ582071 \\
\hline TIMP3 & SULF1 & BCAS1 & SPON1 & GRB7 \\
\hline TK1 & COL8A1 & DEGS2 & ASS1 & RAP1GAP \\
\hline SH3BGRL & POSTN & XBP1 & FLNA & C1S \\
\hline SUSD3 & NBL1 & CRYAB & PKIB & U79293 \\
\hline MIA & DCN & EEF1A2 & SBEM & PRSS8 \\
\hline CPA3 & OGN & SLC39A6 & abParts & C17orf37 \\
\hline PPP1R3C & GJB2 & KRT19 & FLJ42258 & MFAP2 \\
\hline SFRP1 & THBS2 & GALNT6 & CRISPLD2 & TFF1 \\
\hline$A T P 1 B 1$ & ACTA2 & FOXA1 & BAMBI & CA12 \\
\hline SLC40A1 & TBC1D9 & GABRP & SYT13 & TBC1D9 \\
\hline CILP & LOXL2 & NPNT & IGHA2 & CAPS \\
\hline
\end{tabular}

Table 1: Top ranked genes by the Mann Whitney score for each subtype in Figure 4 using CorrML. Some genes are presented in boldface because they are commented in the text or feature in Table 2.

Next we need to determine if the genes listed in Table 1 are consistent with previous findings and if they are biologically relevant. Previously we investigated a number of microarray datasets for breast cancer and the results in Table 1 are consistent with these previous findings. In Carrivick et al [9] we investigated four microarray datasets using a Bayesian variational method [24]. This analysis indicated 4 or 5 principal subtypes of breast cancer. It clearly showed a recognised $E R B B 2+, E S R 1$ - subtype of breast cancer typified by elevated expression of ERBB2 and GRB 7 [27]. A second subtype has a clear connection with the basaloid subtype of breast cancer [27]. Using a variational Bayes method $[1,3]$ we investigated 7 datasets for primary breast carcinoma $([27,29,31]$ and a composite dataset of 614 samples $[13,23,30,33]$ which all used the Affymetrix U133A chip (see [8] for full details). This gave the genetic signature of the basaloid subtype in Table 2 which has a good match to the signature under $C L 5$ in Table 1 above. 


\begin{tabular}{|c|c|c|c|}
\hline Sorlie et al [27] & West et al [31] & Van t' Veer et al [29] & Composite \\
\hline TFF3 & CRIP1 & $V G L L 1$ & FOXA1 \\
\hline XBP1 & XBP1 & AGR2 & AGR2 \\
\hline FOXA1 & FOXA1 & TFF3 & XBP1 \\
\hline GATA3 & $C E B P D$ & ESR1 & $M L P H$ \\
\hline B3GNT5 & HSPAB & CA12 & FLJ20174 \\
\hline GALNT10 & GATA3 & DSC2 & CA12 \\
\hline FBP1 & $R A R A$ & NAT1 & GATA3 \\
\hline DSC2 & CRYAB & $E S T$ & AK127020 \\
\hline FOXC1 & GATA3 & CDH3 & CA12 \\
\hline FOXC1 & FBP1 & FOXC1 & CA12 \\
\hline FLT1 & KRT18 & SCUBE2 & GATA3 \\
\hline FOXC1 & $M S N$ & AR & AR \\
\hline GATA3 & TCEAL1 & Corf7 & TFF3 \\
\hline$S L C 11 A 3$ & SCNN1A & SLC7A2 & $A B A T$ \\
\hline$S L C 11 A 3$ & NSEP1 & GABRP & FBP1 \\
\hline$M G C 27171$ & CDH3 & $E S T$ & DSC2 \\
\hline NAT1 & $B F$ & XPB1 & GATA3 \\
\hline MRPS14 & TFF3 & $B C M P 11$ & CA12 \\
\hline LOC51313 & Hu. clone 23948 & VAV3 & TFF1 \\
\hline$M G C 10710$ & FSCN1 & $E S T$ & GABRP \\
\hline
\end{tabular}

Table 2: The top-ranked genes distinguishing the basaloid subtype of breast cancer. The composite dataset of 614 samples is taken from [13, 23, 30,33], which all use the Affymetrix U133A chip. Repeat gene names in a column derive from multiple probes for that gene.

Furthermore, the genes in Table 1 under $C L 5$ appear biologically significant. The $\mathrm{X}$ box-binding protein, $X B P 1$, is believed to be regulated by FOXA1 [10]. The biological importance of FOXA1 is also apparent from some recent results reported in the literature: a substantial number of estrogen response elements (EREs) have associated binding sites for FOXA1 [10, 18]. Similarly GATA3 has associated co-expression with XPBP1 and ESR1 [17]. We also note that these genes has been previously identified and discussed by other authors [11, 12]. A very similar story emerges if we use the variational Bayes approach outlined in section 2.3. We likewise find a cluster with genes FOXC1, AGR2, FOXA1, GATA3, TFF1, MLPH, XBP1, GABRP ranked in the top 20. Thus the genes highlighted by the method appear to be consistent with previous studies, consistent between the two correspondence algorithms and biologically significant. The advantage of our proposed correspondence models is that we now have additional information about the role of microRNA within given subtypes. In Table 3 we give the top ranked abnormally expressing microRNA within each subtypes as identified by the correspondence model. 


\begin{tabular}{|ll|ll|ll|ll|ll|}
\hline Cl1 & & Cl2 & & Cl3 & & Cl4 & & Cl5 & \\
\hline miR-505 & 0.38 & miR-137 & 0.26 & miR-152 & 1.13 & miR-30b & 0.66 & miR-199a & 0.62 \\
\hline miR-181c & 0.37 & miR-133a & 0.19 & miR-342 & 0.99 & miR-15b & 0.63 & miR-99a & 0.57 \\
\hline miR-142-5p & 0.36 & miR-9 & 0.19 & miR-29a & 0.98 & miR-15a & 0.60 & miR-199b & 0.555 \\
\hline miR-185 & 0.31 & miR-9 & 0.18 & miR-331 & 0.96 & miR-30c & 0.57 & miR-199a & 0.547 \\
\hline miR-203 & 0.31 & miR-18a & 0.08 & miR-214 & 0.95 & miR-195 & 0.55 & miR-214 & 0.474 \\
\hline miR-200a & 0.30 & miR-128b & 0.07 & miR-199b & 0.94 & miR-16 & 0.49 & miR-100 & 0.471 \\
\hline miR-183 & 0.29 & miR-138 & 0.06 & miR-126 & 0.90 & miR-21 & 0.49 & miR-130a & 0.453 \\
\hline miR-509 & 0.29 & miR-211 & 0.03 & miR-145 & 0.89 & miR-20a & 0.45 & miR-382 & 0.429 \\
\hline miR-107 & 0.29 & miR-335 & 0.03 & miR-24 & 0.89 & miR-30a-3p & 0.45 & miR-125b & 0.42 \\
\hline miR-93 & 0.29 & miR-429 & 0.02 & miR-27a & 0.88 & miR-210 & 0.44 & let-7b & 0.40 \\
\hline
\end{tabular}

Table 3: Top ranked microRNA by Mann Whitney score using CorrML.

We also used the Mann-Whitney score to rank microRNA expressions. In Table 3 we give the mean values, $\tilde{\mu}_{h k}$, for the 10 top-ranked microRNA expressions using the CorrML model clusters. As with this Table, a plot of all microRNA expression values, averaged per cluster, indicates substantial differences between the microRNA expression profiles between subtypes. The most aggressive subtype ( $\mathrm{Cl} 3$ in Figure 4) appears to be linked with extensive abnormally high expression of microRNA, followed by $\mathrm{Cl} 4$ and $\mathrm{Cl} 5$ which have small subsets of microRNAs with abnormally high expression.

We also used the Mann-Whitney score to rank microRNA expressions. In Table 3 we give the mean values, $\tilde{\mu}_{h k}$, for the 10 top-ranked microRNA expressions using the CorrML model clusters. As with this Table, a plot of all microRNA expression values, averaged per cluster, indicates substantial differences between the microRNA expression profiles between subtypes. The most aggressive subtype ( $\mathrm{Cl} 3$ in Figure 4) appears to be linked with extensive abnormally high expression of microRNA, followed by $\mathrm{Cl} 4$ and $\mathrm{Cl} 5$ which have small subsets of microRNAs with abnormally high expression. A number of highlighted microRNA appear relevant thus, miR-10b indirectly activates the pro-metastatic gene RHOC by suppressing HOXD10 thus leading to tumor invasion and metastasis [21], miR-214 can induce cell death resistance through targeting the PTEN/Akt pathway [32], miR-21 is oncogenic [25] and let-7 is listed as tumour-suppressive $[20]$.

\section{Conclusion}

In this paper we have introduced a correspondence model for unsupervised learning with multiple types of data. Using a predictive likelihood estimate or a free energy term we can find the appropriate number of clusters in the data. The proposed methods can handle missing values. In Sections 3.1 we argued that cluster analysis on an amalgamated dataset gave inferior perfor- 
mance compared to the proposed models. In Section 3.2 we gave an extended discussion of an application to breast cancer biology: the results for the correspondence model appeared consistent with previous findings and biologically plausible. Furthermore, by incorporating microRNA expression data in addition to gene expression data, the model may give possible new insights into dysregulation of microRNA expression associated with individual breast cancer subtypes. The methods proposed here can be extended in various ways. Firstly, we have presented them for two types of data which are of the same type: continuous valued data (e.g. gene expression data) which can be approximately modelled using a Gaussian. However, we could equally well use discrete data and a multinomial or Poisson distribution to model one or both types of data. We could, of course, also use a variety of MCMC methods. MCMC proved too computationally intensive for determining the model complexity with the large expression array datasets here. However, for smaller datasets, MCMC could be usefully deployed.

Acknowledgements: we thank Andrew Teschendorff (University of Cambridge) for assistance with sourcing and interpreting the breast cancer data of section 3.2. We also thank Simon Rogers, Mark Girolami and Luke Carrivick for discussions.

\section{References}

[1] H Attias. Inferring parameters and structure of latent variable models by variational Bayes. In Proceedings of the 15th Conference on Uncertainty in Artifical Intelligence, pages 21-30. Morgan-Kaufmann, San Francisco, CA, 1999.

[2] H. Attias. A variational bayesian framework for graphical models, 2000.

[3] M J Beal and Z Ghahramani. The variational bayesian em algorithm for incomplete data: with application to scoring graphical model structures. Bayesian Statistics, 7:453-464, 2003.

[4] J Bi, T Zhang, and K Bennett. Column-generation boosting mthods for mixture of kernels. In Proceedings of SIGKDD International Conference on Knowledge Discovery and Data Mining, 2004.

[5] David M. Blei and Michael I. Jordan. Modeling annotated data. In SIGIR '03: Proceedings of the 26th annual international ACM SIGIR conference on Research and development in informaion retrieval, pages 127-134, New York, NY, USA, 2003. ACM Press.

[6] David M. Blei, Andrew Y. Ng, and Michael I. Jordan. Latent dirichlet allocation. Journal of Machine Learning Research, 3:993-1022, 2003.

[7] C Blenkiron et al. MicroRNA expression profiling of human breast cancer identifies new markers of tumour subtype. Genome Biology, 8:R214.1-R214.16, 2007. 
[8] L Carrivick. Probabilistic models in the biomedical sciences. PhD Thesis, Department of Engineering Mathematics, University of Bristol, UK, 2006.

[9] L. Carrivick, S. Rogers, J. Clark, C. Campbell, M. Girolami, and C. Cooper. Identification of prognostic signatures in breast cancer microarray data using bayesian techniques. Journal of the Royal Society: Interface, 3:367-381, 2006.

[10] J Carroll et al. Chromosome-wide mapping of estrogen receptor binding reveals long-range regulation requiring the forkhead protein FOXA1. Cell, 122:33-43, 2005.

[11] A Dobra, B Jones, C Hans, J Nevins, and M West. Sparse graphical models for exploring gene expression data. Journal of Multivariate Analysis, 90:196-212, 2004.

[12] A Dobra and M West. Graphical model-based gene clustering and metagene expression analysis. Technical report, 2004.

[13] P Farmer et al. Identification of molecular apocrine breast tumours by microarray analysis. Oncogene, 24:4660-4671, 2005.

[14] M Girolami and M Zhong. Data integration for classification problems employing gaussian process priors. In Twentieth Annual Conference on Neural Information Processing Systems, 2007.

[15] Mark Girolami and Simon Rogers. Hierarchic bayesian models for kernel learning. In ICML: 22nd International Conference on Machine Learning, Bonn, Germany., August 2005.

[16] M Jordan, Z Ghahramani, T Jaakola, and L Saul. An introduction to variational methods for graphical models. Machine Learning, 37:183-233, 1999.

[17] M Lacroix and G Leclerq. About GATA3, HNF3A and XBP1, three genes co-expressed with the oestrogen receptor-alpha gene (ESR1) in breast cancer. Molecular and Cellular Endocrinology, 219:1-7, 2004.

[18] J Laganiere et al. Location analysis of estrogen receptor $\alpha$ target promoters reveals that FOXA1 defines a domain of the estrogen response. Proceedings National Academy Sciences, 102:11651-11656, 2005.

[19] G R G Lanckriet, T De Bie, N Cristianini, M I Jordan, and W S Noble. A statistical framework for genomic data fusion. Bioinformatics, 20:2626-2635, 2004.

[20] Y S Lee and A Dutta. The tumor suppressor microrna let-7 represses the hmga2 oncogene. Genes and Development, 21:1025-1030, 2007.

[21] M Li, J Teruya-Feldstein, and R A Weinberg. Tumour invasion and metastasis initiated by microrna-10b in breast cancer. Nature, 449:682-688, 2007. 
[22] M Middendorf, A Kundaje, C Wiggins, Y Freund, and C Leslie. Predicting genetic regulatory response using classification. In Proceedings of the Twelfth International Conference on Intelligent Systems in Molecular Biology (ISMB 2004), page in press, 2004.

[23] Y Pawitan et al. Gene expression profiling spares early breast cancer patients from adjuvant therapy: derived and validated in two population-based cohorts. Breast Cancer Research, 7:R953-R964, 2005.

[24] S Rogers, M Girolami, C Campbell, and R Breitling. The latent process decomposition of cDNA microarray datasets. IEEE/ACM Transactions on Computational Biology and Bioinformatics, 2:143-156, 2005.

[25] M-L Si et al. mir-21-mediated tumor growth. Oncogene, 26:2799-2803, 2007.

[26] S Sonnenburg et al. Large scale multiple kernel learning. Journal of Machine Learning Research, 7:1531-1565, 2006.

[27] T Sorlie et al. Gene expression patterns of breast carcinomas distinguish tumor subclasses with clinical implications. Proceedings National Academy Sciences, 98:10869-10874, 2001.

[28] A Teschendorff et al. A variational bayesian mixture modelling framework for cluster analysis of gene-expression data. Bioinformatics, 21:3025-3033, 2005.

[29] L van 't Veer et al. Gene expression profiling predicts clinical outcome of breast cancer. Nature, 415:530-535, 2002.

[30] Y Wang et al. Gene-expression profiles to predict distant metastasis of lymph-node-negative primary breast cancer. The Lancet, 365:671-679, 2005.

[31] M West et al. Predicting the clinical status of human breast cancer using gene expression profiles. Proceedings of the National Academy of Sciences, 98:11462-11467, 2001.

[32] Hua Y et al. Microrna expression profiling in human ovarian cancer: mir-214 induces cell survival and cisplatin resistance by targeting pten. Cancer Research, 68:425-433, 2008.

[33] F Yang et al. Laser microdissection and microarray analysis of breast tumors reveal er- $\alpha$ related genes and pathways. Oncogene, 25:1413-1419, 2006. 


\section{Appendices}

\section{A Lower Bound and Predictive Likelihood for CorrML}

In this appendix we outline the computation of the KL-divergence and predictive likelihood for the first correspondence model, $\operatorname{Corr} M L$.

\section{Lower bound (negative KL-divergence):}

The lower bound for the log likelihood (denoted $\mathcal{L}$ ) equals the negative KL-divergence:

$$
\mathcal{L}=\int \sum_{z, y} q(z, y, \theta) \log \frac{p(C, E, z, y, \theta \mid \Theta)}{q(z, y, \theta)} d \theta=-\mathrm{KL}(q(\theta, z, y) \| p(\theta, z, y \mid E, C, \Theta)) .
$$

Estimation of the log joint distribution gives:

$$
\begin{aligned}
\mathcal{L}= & D\left[\log \Gamma\left(\sum_{k} \alpha_{k}\right)-\sum_{k} \log \Gamma\left(\alpha_{k}\right)\right]-\sum_{d}\left[\log \Gamma\left(\sum_{k} \gamma_{d k}\right)-\sum_{k} \log \Gamma\left(\gamma_{d k}\right)\right] \\
& +\sum_{d, k}\left[\left(\alpha_{k}-\gamma_{d k}+\sum_{h} R_{d h k}\right)\left(\psi\left(\gamma_{d k}\right)-\psi\left(\sum_{j} \gamma_{d j}\right)\right)\right] \\
& +\sum_{d, h, k}\left[R_{d h k}\left(\log \mathcal{N}\left(c_{h d} \mid \tilde{\mu}_{h k}, \tilde{\sigma}_{h k}^{2}\right)-\log R_{d h k}\right)\right] \\
& +\sum_{d, g, h} Q_{d g h}\left[\left(\sum_{k} R_{d h k} \log \mathcal{N}\left(e_{g d} \mid \mu_{g k}, \sigma_{g k}^{2}\right)\right)-\log Q_{d g h}\right] .
\end{aligned}
$$

\section{Predictive likelihood:}

First, marginalizing the joint probability given by equation (1) with respective to $z$ gives

$$
\begin{aligned}
& p(C, E, y \mid \Theta)=\prod_{d} \int_{\theta_{d}} \sum_{z} p\left(C_{d}, E_{d}, z_{d}, y_{d}, \theta_{d} \mid \Theta\right) d \theta_{d} \\
& =\prod_{d} \int_{\theta_{d}} \prod_{h}\left[\sum_{k} \theta_{d k} \mathcal{N}\left(C_{h d} \mid \tilde{\mu}_{h k}, \tilde{\sigma}_{h k}^{2}\right) \prod_{g}\left(\frac{1}{H} \mathcal{N}\left(E_{g d} \mid \mu_{g k}, \sigma_{g k}^{2}\right)\right)^{y_{d g}, h}\right] d \theta .
\end{aligned}
$$

However, further marginalizing with respect to $y$ will lead to very intensive computation since the dimension of expression gene $g$ is usually large. Hence, we are forced to consider approximation of the test likelihood. To this end, we replace the untractable term $\prod_{g}\left(\frac{1}{H} \mathcal{N}\left(E_{g d} \mid \mu_{g k}, \sigma_{g k}^{2}\right)\right)^{y_{d g, h}}$ by its average

$$
\prod_{g}\left(\frac{1}{H} \mathcal{N}\left(E_{g d} \mid \mu_{g k}, \sigma_{g k}^{2}\right)\right)
$$

Consequently, we have the following approximation to the likelihood 


$$
p(C, E \mid \Theta) \approx \prod_{d} \int_{\theta_{d}} \prod_{h}\left[\sum_{k} \theta_{d k} \mathcal{N}\left(C_{h d} \mid \tilde{\mu}_{h k}, \tilde{\sigma}_{h k}^{2}\right) \prod_{g}\left(\frac{1}{H} \mathcal{N}\left(E_{g d} \mid \mu_{g k}, \sigma_{g k}^{2}\right)\right)\right] d \theta
$$

The integral with respect to $\theta$ can be further approximated by a sampling method described in Blei and Jordan [5].

\section{B Updates for CorrVB}

We now list the updates equations for the variational Bayesian inference for the correspondence model.

- For $\theta$, we have that $q(\theta \mid \gamma) \propto \exp \left(\mathbf{E}_{q(z, y, \Xi)}[\log p(C, E, \theta, z, y, \Xi \mid \alpha)]\right)$ which yields that

$$
\gamma_{d k}=\alpha_{k}+\sum_{h} R_{d h k}
$$

- For $z$, we have that $q(z \mid R)=\prod_{d, h} q\left(z_{d h} \mid R_{d h}\right) \propto \exp \left(\mathbf{E}_{q(\theta, y, \Xi)}[\log p(C, E, \theta, z, y, \Xi \mid \alpha)]\right)$. Consequently

$$
\begin{aligned}
& R_{d h, k} \propto \exp \left[\psi(d k)-\psi\left(\sum_{k} \gamma_{d k}\right)+\frac{1}{2}\left(\psi\left(a_{h k}\right)+\log b_{h k}\right)-\frac{1}{2}\left(\left(C_{d h}-m_{h k}\right)^{2}+\frac{1}{v_{h k}}\right) a_{h k} b_{h k}\right. \\
& \left.+\frac{1}{2} \sum_{g} Q_{d g h} \psi\left(a_{g k}\right)+\log b_{g k}-a_{g k} b_{g k}\left(\left(E_{d g}-m_{g k}\right)^{2}+\frac{1}{v_{g k}}\right)\right]
\end{aligned}
$$

with the normalization $\sum_{k} R_{d h, k}=1$ for any $d, h$.

- For the latent variable $y$, from the equation

$$
q(y \mid Q)=\prod_{d, g} q\left(y_{d g} \mid Q_{d g}\right) \propto \exp \left(\mathbf{E}_{q(\theta, z, y, \Xi)}[\log p(C, E, \theta, z, y, \Xi \mid \alpha)]\right)
$$

we know that

$$
Q_{d g, h} \propto \exp \left[\log \phi_{g h}+\frac{1}{2} \sum_{k} R_{d h k}\left(\psi\left(a_{g k}\right)+\log b_{g k}-\frac{a_{g k} b_{g k}}{v_{g k}}\left(\left(E_{d g}-m_{g k}\right)^{2}\right)\right)\right]
$$

with the normalization $\sum_{h} Q_{d g, h}=1$ for any $d, g$.

- For the latent variables $\widetilde{\mu}$ and $\widetilde{\beta}$, we have that

$$
\left.q(\widetilde{\mu} \mid \widetilde{m}, \widetilde{v})=\prod_{h, k} \mathcal{N}\left(\widetilde{\mu}_{h k} \mid \widetilde{m}_{h k}, \widetilde{v}_{h k}\right) \propto \exp \left(\mathbf{E}_{q(\theta, z, y, \mu, \beta, \widetilde{\beta}}\right)[\log p(C, E, \theta, z, y, \Xi \mid \alpha)]\right),
$$


and and

$$
q(\widetilde{\beta} \mid \widetilde{a}, \widetilde{b})=\prod_{h, k} \Gamma\left(\widetilde{\beta}_{h k} \mid \widetilde{a}_{h k}, \widetilde{b}_{h k}\right) \propto \exp \left(\mathbf{E}_{q(\theta, z, y, \mu, \beta, \widetilde{\mu})}[\log p(C, E, \theta, z, y, \Xi \mid \alpha)]\right) .
$$

Consequently,

$$
\begin{gathered}
\widetilde{m}_{h k}=\frac{m_{0} v_{0}+\left(\sum_{d} C_{d h} R_{d h k}\right) \widetilde{a}_{h k} \widetilde{b}_{h k}}{\widetilde{v}_{h k}}, \quad \widetilde{v}_{h k}=v_{0}+\widetilde{a}_{h k} \widetilde{b}_{h k} \sum_{d} R_{d h k} \\
\widetilde{a}_{h k}=a_{0}+\frac{1}{2} \sum_{d} R_{d h k}, \quad \widetilde{b}_{h k}^{-1}=\frac{1}{b_{0}}+\frac{1}{2} \sum_{d} R_{d h k}\left[\left(C_{d h}-\widetilde{m}_{h k}\right)^{2}+\frac{1}{\widetilde{v}_{h k}}\right]
\end{gathered}
$$

- For $\mu$ and $\beta$ we have that

$$
q(\mu \mid m, v)=\prod_{g, k} \mathcal{N}\left(\mu_{g k} \mid m_{g k}, v_{g k}\right) \propto \exp \left(\mathbf{E}_{q(\theta, z, y, \beta, \widetilde{\mu}, \widetilde{\beta})}[\log p(C, E, \theta, z, y, \Xi \mid \alpha)]\right),
$$

and

$$
q(\beta \mid a, b)=\prod_{g, k} \Gamma\left(\beta_{g k} \mid a_{g k}, b_{g k}\right) \propto \exp \left(\mathbf{E}_{q(\theta, z, y, \mu, \widetilde{\mu}, \widetilde{\beta})}[\log p(C, E, \theta, z, y, \Xi \mid \alpha)]\right),
$$

Consequently,

$$
m_{g k}=\frac{m_{0} v_{0}+\left(\sum_{d, h} Q_{d g, h} R_{d h, k}\right) a_{h k} b_{h k}}{v_{g k}}, \quad v_{g k}=v_{0}+a_{g k} b_{g k} \sum_{d, h} Q_{d g, h} R_{d h, k}
$$

and

$$
a_{g k}=a_{0}+\frac{1}{2} \sum_{d, h} Q_{d g, h} R_{d h, k}, \quad b_{g k}^{-1}=\frac{1}{b_{0}}+\frac{1}{2} \sum_{d, h} Q_{d g, h} R_{d h, k}\left[\left(E_{d g}-m_{g k}\right)^{2}+\frac{1}{v_{g k}}\right]
$$

In MAP of type II, we also update the Dirichlet parameter by the following equation

$$
\hat{\alpha}=\arg \max \left[D\left(\ln \Gamma\left(\sum_{k} \alpha_{k}\right)-\sum_{k} \ln \Gamma\left(\alpha_{k}\right)+\sum_{d, k}\left(\alpha_{k}-1\right)\left(\psi\left(\gamma_{d k}\right)-\psi\left(\sum_{k} \gamma_{d k}\right)\right)\right)\right]
$$




\section{A Joint Mixture Model}

For the joint mixture model (JMM) mentioned in Section 3.1, the functional relationship between the different data sets is modelled via a jointly clustering Dirichlet distribution. Samples in the different data sets are generated separately. This model is described as follows:

\section{For a fixed data index $d$ for both $E(G \times D$ matrix) and $C(H \times D$ matrix $)$}

1. Prior distributions: $\theta_{d} \sim \operatorname{Dir}_{K}(\alpha)$

2. Generate $C_{d}$ :

(a) Choose process for $C_{h d}$ : $\quad \tilde{z}_{d h} \sim \operatorname{Multi}\left(\theta_{d}\right)$

(b) Sample $C_{h d} \sim \mathcal{N}\left(C_{h d} \mid \tilde{\mu}_{h \tilde{z}_{d h}}, \tilde{\sigma}_{h \tilde{z}_{d h}}\right)$ where $\mathcal{N}\left(C_{h d} \mid \tilde{\mu}, \tilde{\sigma}^{2}\right)$ denotes a normal distribution with mean $\tilde{\mu}$ and variance $\tilde{\sigma}^{2}$.

3. Generate $E_{d}$ :

(a) Choose process for $E_{g d}$ : $\quad z_{d g} \sim \operatorname{Multi}\left(\theta_{d}\right)$

(b) Sample $E_{g d} \sim \mathcal{N}\left(E_{g d} \mid \mu, \sigma, z\right)=\mathcal{N}\left(E_{g d} \mid \mu_{g z_{d g}}, \tilde{\sigma}_{g z_{d g}}^{2}\right)$

Using the notation $\Theta=\{\alpha, \mu, \sigma, \tilde{\mu}, \tilde{\sigma}\}$, the joint distribution for a given data index $d$ is given by:

$$
\begin{aligned}
p\left(C_{d}, E_{d}, \tilde{z}_{d}, z_{d}, y_{d}, \theta_{d} \mid \Theta\right) & =p\left(\theta_{d} \mid \alpha\right) \prod_{h}\left[p\left(\tilde{z}_{d h} \mid \theta_{d}\right) \mathcal{N}\left(C_{h d} \mid \tilde{\mu}_{h z_{d h}}, \tilde{\sigma}_{h z_{d h}}^{2}\right)\right] \\
& \times \prod_{g} p\left(z_{d g} \mid \theta_{d}\right) \mathcal{N}\left(E_{g d} \mid \mu_{g z_{d g}}, \tilde{\sigma}_{g z_{d g}}^{2}\right)
\end{aligned}
$$

The overall joint distribution is then given by:

$$
p(C, E, \tilde{z}, z, y, \theta \mid \Theta)=\prod_{d} p\left(C_{d}, E_{d}, \tilde{z}_{d}, z_{d}, y_{d}, \theta_{d} \mid \Theta\right)
$$

